\title{
Social and Cognitive Strategies for Coping With Accountability: Conformity, Complexity, and Bolstering
}

\author{
Philip E. Tetlock and Linda Skitka \\ University of California, Berkeley \\ Richard Boettger \\ Texas Christian University
}

\begin{abstract}
This experiment tested predictions derived from a social contingency model of judgment and choice that identifies 3 distinctive strategies that people rely on in dealing with demands for accountability from important interpersonal or institutional audiences. The model predicts that (a) when people know the views of the audience and are unconstrained by past commitments, they will rely on the low-effort acceptability heuristic and simply shift their views toward those of the prospective audience, (b) when people do not know the views of the audience and are unconstrained by past commitments, they will be motivated to think in relatively flexible, multidimensional ways (preemptive selfcriticism), and (c) when people are accountable for positions to which they feel committed, they will devote the majority of their mental effort to justifying those positions (defensive bolstering). The experiment yielded results supportive of these 3 predictions. The study also revealed some evidence of individual differences in social and cognitive strategies for coping with accountability.
\end{abstract}

Many writers have criticized cognitive social psychology for its apparent indifference to the interpersonal and institutional settings within which people make judgments and choices (e.g., Gergen, 1982; Sampson, 1981). Although these critiques make important points, it is necessary to advance beyond metatheoretical position papers and to specify the particular ways in which interpersonal and institutional variables interact with cognitive tendencies of the perceiver to shape how people actually make up their minds.

Recent research on accountability points to one possible answer. Tetlock (1985a) has argued that accountability is a universal feature of everyday decision-making environments. Accountability, from this standpoint, is a critical rule- and normenforcement mechanism: the social psychological link between individual thinkers on the one hand and the social systems to which they belong on the other. The fact that people are ultimately accountable for their decisions is an implicit or explicit constraint on virtually everything they do. Failure to behave in ways for which one can construct acceptable accounts leads to varying degrees of censure-depending, of course, on the gravity of the offense and the norms of the organization (Schlenker, 1982; Scott \& Lyman, 1968; Tetlock, 1981a).

A good deal of experimental evidence indicates that accountability pressures can affect both what people think (the beliefs and preferences they express) and how they think (the reasoning

This research was supported by National Institute of Mental Health Grant 39942.

We appreciate the helpful comments of Associate Editor Norbert Kerr and three anonymous but integratively complex reviewers.

Correspondence concerning this article should be addressed to Philip E. Tetlock, Department of Psychology, 3210 Tolman Hall, University of California, Berkeley, California 94720. strategies that underlie those beliefs and preferences). The evidence, however, is-at least superficially-inconsistent and confusing.

Some strands of research suggest that people cope with accountability by simply seeking out the most expedient or obviously acceptable position. For instance, negotiators who expect to justify bargaining outcomes to the constituencies they represent have more difficulty reaching mutually acceptable compromise agreements than do negotiators who are not under such pressure (e.g., Pruitt, 1981). The most plausible explanation is that accountability to constituents (who favor tough negotiating stands) induces concern for appearing strong by refusing to make concessions. Negotiators respond by using competitive tactics that create obstacles to resolving conflicts of interest, but that protect their social identities in the eyes of constituents. Experimental work on ingratiation (Jones \& Wortman, 1973) and strategic attitude shifts (Cialdini, Levy, Herman, \& Evenbeck, 1973) points to a similar conclusion. People view anticipatory opinion conformity as a reliable means of avoiding the disapproval and perhaps gaining the approval of others (cf. Tetlock, 1983a).

Other strands of research suggest that people cope with accountability by thinking in flexible, multidimensional ways that reduce or even eliminate well-replicated judgmental biases. Tetlock, for instance, has conducted a series of studies in which experimental manipulations of accountability (expecting to justify one's views) have (a) reduced primacy effects in an impression-formation paradigm by increasing willingness to pay attention to all the evidence and to modify initial impressions in response to contradictory evidence (Tetlock, 1983b), (b) reduced overattribution effects in an essay-attribution paradigm by increasing sensitivity to the situational pressures on others (Tetlock, 1985b), and (c) reduced overconfidence effects in a personality-prediction paradigm by increasing awareness of 
complexities in the behavior of others (Tetlock \& Kim, 1987). Other investigators have reported similar results (e.g., Cvetkovich, 1978; Hagafors \& Brehmer, 1983; Rozelle \& Baxter, 1981).

A third line of empirical activity suggests that accountability motivates people to be defensive-to think of reasons why they are right and potential critics are wrong. Both impression management and cognitive dissonance researchers have repeatedly documented the tendency for people to justify their conduct, especially when that conduct casts doubt on their competence or integrity and is public knowledge (for reviews, see Tetlock \& Manstead, 1985; Wicklund \& Brehm, 1976). Fox and Staw (1979) provided an interesting experimental demonstration of how accountability can exacerbate this process. Decision makers were most likely to increase their commitment of resources to a failing policy when they were most vulnerable (low job security) and were accountable to a skeptical board of directors. Accountability here led to simplistic and self-serving bolstering of policies to which subjects felt personally committed. Accountable subjects had a harder time than unaccountable subjects in writing off "sunk costs" and in acknowledging that they had made a mistake.

The key theoretical question is obviously "Under what conditions should we expect one or another of these qualitatively different coping responses to accountability to emerge?" Tetlock (1985a) proposed a social contingency model of judgment and choice that both integrates available evidence into a comprehensive framework and leads to novel testable predictions. The model assumes that people tend to be "cognitive misers" who rely on simple, low-effort heuristics that allow them to make up their minds quickly, easily, and with confidence in their judgments (cf. Fiske \& Taylor, 1984). When people know the views of the audience to whom they are accountable and are unconstrained by past commitments, the cognitive miserly response is the acceptability heuristic (Tetlock, 1985a, p. 311)to avoid unnecessary cognitive work (analyzing and balancing the pros and cons of different positions) and adopt the salient, socially acceptable position. When people do not know the views of the audience and are unconstrained by past commitments, this relatively effortless option is foreclosed. Here people can be motivated to be complex, self-critical information processors who try to anticipate the objections of potential critics-a process of preemptive self-criticism (Tetlock, 1983a). Finally, when people feel accountable for positions to which they are already committed, the coping strategies of conformity and preemptive self-criticism lose much of their attraction. People do not want to appear to lack the courage of their convictions. Hence, the majority of mental effort goes into generating thoughts that justify their original commitments-an exercise in retrospective rationality (Staw, 1980).

These coping strategies are not, of course, mutually exclusive. People may rely on composite or hybrid strategies. For instance, people might respond to accountability pressures from an unknown audience by shifting their public positions toward their best guess of the audience's position and by engaging in preemptive self-criticism. The result would be a complex, middleground position. Alternatively, people might respond to accountability to a known audience by shifting their public posi- tions toward the audience and by actually integrating the point of view of the audience into their own private position.

The experiment reported here tests the central hypotheses of the social contingency model. The study takes the form of a mixed, 2 (attitudes first vs. thoughts first) $\times 4$ (unaccountable vs. accountable-unknown vs. accountable-liberal vs. accountable-conservative) $\times 4$ (type of issue) design. Subjects either did or did not expect to justify their policy preferences on four controversial issues. Accountable subjects either did not know the views of the audience to whom they were accountable or did know of those views and expected them to be liberal or conservative in nature. Finally, subjects were given the opportunity to organize and write down their private thoughts on the issues either before or after they committed themselves to stands. The key dependent variables were the liberalism-conservatism of the attitudes that subjects expressed on the policy issues and the content and complexity of the private thoughts that subjects reported on each issue.

\section{Theoretical Expectations}

Our theoretical expectations were as follows:

Strategic attitude shifts. If subjects truly are cognitive misers who seek low-effort solutions to the social predicament created by the experiment, they will rely on the acceptability heuristic and shift their attitudes toward those of the anticipated audience. It should not matter, moreover, whether subjects have an extended opportunity to think about the issues before taking a stand. The term acceptability heuristic implies that strategic attitude shifting is a highly overlearned, even automatic, response that requires minimal conscious monitoring (cf. Sherman \& Corty, 1984). This argument leads to the prediction of a main effect (regardless of the timing of thought listing) for subjects to report more liberal thoughts to a liberal audience and more conservative thoughts to a conservative audience.

The term heuristic may, however, be misleading. Attitude shifting may also be the product of a more cognitively demanding process of role- or perspective-taking. Subjects who anticipate justifying their views to a known audience may-in anticipation of the conversation-try to imagine how that person thinks. This cognitive preparation for the conversation will have the net effect of making liberal or conservative thoughts more salient or cognitively available, which, in turn, may influence the subjects' self-perceptions of their own positions. From this standpoint, cognitive responses at least partly mediate strategic attitude shifts. Subjects will be much more likely to shift their attitudes toward those of a liberal or conservative audience when they have had an opportunity to organize and write down their thoughts before as opposed to after taking stands on the issues.

Preemptive self-criticism. Strategic attitude shifting is a less viable coping strategy when the views of the prospective audience are unknown. Here cognitive preparation for the conversation will largely take the form of preemptive self-criticism. Subjects will try to anticipate the counterarguments that others might raise and to construct plausible defenses against those objections. Accordingly, we expect the most integratively complex and evaluatively inconsistent thoughts when subjects have an opportunity to organize their thoughts before taking attitudi- 
nal stands and when subjects are accountable to an audience with unknown views.

Just as subjects in the accountable-to-known-audience conditions may be more thoughtful than the social contingency model suggests, it is also possible that subjects in the accountable-unknown conditions may be less thoughtful than the model suggests. In lieu of thinking carefully about the issues, these subjects may simply move toward the midpoints of the attitude scales-a low-effort means of minimizing sharp disagreements with the prospective audience.

Defensive bolstering. The social contingency model maintains that once people have committed themselves to a position, a major function of thought becomes the justification of that position. Two specific predictions follow from the model. First, reported thoughts will tend to be more evaluatively consistent and less integratively complex when subjects report their thoughts after making an attitudinal commitment, regardless of accountability. Second, this tendency toward simplification should be more pronounced among accountable subjects (a Timing $\times$ Accountability interaction). Only intrapsychic pressures (e.g., dissonance reduction) motivate simplification of thought when attitudes-first subjects are not accountable; a combination of intrapsychic and impression management pressures motivate simplification when attitudes-first subjects are accountable.

As an ancillary goal, the study also explores individual differences in coping responses to accountability. We explore the possibility that subjects who are predisposed to think in rigid, dichotomous terms (high scorers on Rokeach's, 1960, Dogmatism scale) are more likely to rely on defensive bolstering and less likely to rely on preemptive self-criticism. We also explore whether subjects who are especially concerned with their public images (high scorers on Social Anxiety and Public SelfConsciousness scales) are more likely to rely on strategic attitude shifts as a coping strategy.

\section{Method}

\section{Subjects}

We randomly assigned 325 subjects to one of eight conditions in a 2 (timing of measures) $\times 4$ (type of accountability) $\times 4$ (type of issuerepeated measure) design. Subjects took part in the experiment in small groups.

\section{Procedure}

The experimenter began each session by explaining that the objective of the study was to investigate how people think about controversial public policy issues. The experimenter also provided definitions of four key political concepts that the subjects would be encountering during the study: (a) affirmative action quotas (quotas that guarantee that a certain percentage of the people admitted to educational institutions or hired for certain jobs come from designated minority groups), (b) tuition at the University of California (subjects had to be informed that there was no tuition at the university - that their fees pay for "special services" such as sports facilities and student health services; tuition requires that students also pay for the costs of instruction, which would mean a substantial increase in fees), (c) the nuclear freeze (a policy that would require the United States and the Soviet Union to stop manufac- turing and deploying new nuclear weapons), and (d) capital punishment (support for the death penalty for particularly heinous or brutal crimes).

Thought assessment first. After these preliminaries, subjects received one of four sets of instructions. One group (the unaccountable condition) was told the following:

\begin{abstract}
We want you to write down your thoughts and feelings for each issue. You should understand that everything you write down will be completely confidential and not traceable to you personally. In fact, the responses you make will not even be analyzed by researchers at this university. The data will be shipped to researchers at another institution who specialize in analyzing these types of "free response" materials. Please respond with complete candor and honesty in order to ensure the success of the project. We need to know what you think when you don't have to worry about how other people will react to your views. Do not sign your name on the thought protocol, but instead use a five-digit number of your own choosing on top of each page. Please use the same code identification on each page of the questionnaire so we can keep your responses together and preserve your confidentiality.
\end{abstract}

A second group (the accountable-unknown condition) was given the same instructions plus the following information:

To help us better understand the interpersonal communication of attitudes, in the communication phase of the experiment you will be asked to explain and justify your opinions to another subject. This discussion will be audiotaped to facilitate analysis of the communication process.

The third and fourth groups (the accountable-liberal and accountable-conservative conditions) learned more about the person to whom they would be talking in the communication phase of the experiment. Those in the accountable-liberal condition learned that they would be asked to explain and justify their opinions to another subject who (it was known from an earlier testing session) held consistently liberal views on major social issues and supported policies such as affirmative action and the nuclear freeze and opposed capital punishment and university tuition.

Those in the accountable-conservative condition expected to explain and justify their opinions to another subject who held consistently conservative views and took exactly the opposite stands on the issues.

Subjects were then given 5 min to list all their thoughts and feelings about each of the four issues: affirmative action, university tuition, the nuclear freeze, and capital punishment (presented in one of six randomly determined orders). After completing the thought protocols, subjects responded to the attitude scales. Subjects in the accountability conditions were told that, since they had had a chance to think about the issues they would be discussing, we now wanted them to express their policy preferences on a series of attitude scales. Subjects were also told that their responses to these scales would be the focus of the conversation during the communication phase of the experiment, and would be provided to their discussion partner. Subjects in the unaccountable condition were reminded that all responses would be absolutely anonymous and confidential.

The attitude measure for each policy issue consisted of three 7-point Semantic Differential scales designed to tap the evaluative dimension of meaning: unfair-fair, foolish-wise, and bad-good. On completing the attitude scales, subjects provided some additional demographic information. In addition, subjects in the three accountability conditions were asked to sign their names (ostensibly to facilitate familiarity with their discussion partners and to make matching easier, but actually to enhance their sense of personal accountability for their opinions).

Attitude assessment first. In these conditions, subjects received essentially the same instructions as in thoughts-first conditions with one key exception. Like thoughts-first subjects, attitudes-first subjects learned whether they were accountable before adopting an attitudinal stand. 
Subjects in the latter group, however, were given an opportunity to write down their thoughts on each issue only after they had committed themselves to attitudinal stands.

Subject reactions to experiment. After subjects had completed the attitude and thought protocol measures, we assessed their perceptions of, and emotional reactions to, the experiment. Subjects responded to all the statements asked on a scale ranging from 1 (not at all true) to 7 (extremely true). This questionnaire included statements such as "I am feeling nervous," "I am feeling embarrassed," "I am looking forward to the rest of the experiment," "I am having fun," "I like this experiment because it is giving me an opportunity to express my views," and "I am feeling uncomfortable with this experiment because it is requiring me to disclose my views." Subjects also completed manipulation-check questions (e.g., Did subjects believe that they would be talking to someone else, and if so, with whom did they expect to talk? What were their feelings about the prospective conversation?).

Additional data collected. All subjects also completed a series of personality measures, including a Dogmatism scale (Rokeach, 1960) and scales designed to assess private and public self-consciousness and social anxiety (Buss, 1980).

Integrative complexity coding. The thoughts that subjects reported on each issue were coded for integrative complexity. This coding system was originally developed for scoring responses to a semiprojective test designed to assess individual differences in cognitive style (Schroder, Driver, \& Streufert, 1967). More recent work has demonstrated the coding system to be sensitive to situational determinants of integrative complexity (e.g., role demands and groupthink) as well as individual differences in this characteristic (Tetlock, 1979, 1981b, 1983a, 1983b, 1984, 1985a, 1985b, 1986; Tetlock \& Boettger, 1989; Tetlock, Hannum, \& Micheletti, 1984).

Integrative complexity is defined in terms of two cognitive structural properties: differentiation and integration. Differentiation refers to the number of evaluatively distinct dimensions of a problem that an individual takes into account. For instance, an individual might take an undifferentiated view of capital punishment by focusing only on the need to deter murder or only on the immorality of taking human life. A more differentiated approach would recognize at least two different perspectives on the issue (e.g., the need to deter murder and to avoid executing innocent persons). Integration refers to the development of complex connections among differentiated characteristics. (Differentiation is therefore a necessary condition for integration.) The complexity of integration depends on whether the individual perceives the differentiated characteristics in isolation (low integration), in simple interactions (moderate integration), or in multiple, contingent patterns (high integration).

The following examples of actual responses by subjects illustrate different levels of integrative complexity:

Score 1 (low differentiation-low integration). Capital punishment is a barbaric practice. A civilized society does not deliberately take the lives of its citizens to avenge crimes. It makes no difference how evil the crime. The basic rule still stands: "Thou shalt not kill." I don't know why some people have such a hard time understanding this.

Score 3 (moderate to high differentiation-low integration). I'm of two minds on this issue. Some crimes are so disgusting that they cry out for the ultimate penalty. But I'm also worried about executing an innocent person. Our courts are not perfect.

Score 5 (moderate to high differentiation-moderate integration). I can see why people disagree on capital punishment. A lot depends on whether you look at the issue from the point of view of the victims or from the point of view of the criminals. Victims want revenge. Criminals want leniency-and leniency may be justified sometimes depending on the circumstances. On balance, I support the death penalty, but only when there is no doubt regarding guilt and only when the crime is especially vicious.
Scores of 7 reflect high differentiation and high integration. No thought protocols were assigned this highest score. Scores at this level require awareness of complex value trade-offs or of complex relationships between different ways of looking at the world. Scores of 2, 4, and 6 represent transition points between adjacent levels.

An important aspect of integrative complexity coding is its focus on structure rather than content. One can be simple or complex in advocating a variety of positions. One's integrative complexity is determined not by the specific positions one endorses, but by the conceptual structure underlying those positions.

Thought protocols were coded for integrative complexity by two trained scorers, one of whom was unaware of the hypotheses and experimental conditions from which the protocols were drawn. Reasonably high agreement existed between coders $(r=.87)$. Disagreements were resolved by using the score assigned by the "double-blinded" coder.

Constructing the balance index. Two judges (both unaware of the sources of the thoughts and the experimental hypotheses) classified the thoughts subjects listed as liberal (pro-affirmative action, pro-nuclear freeze, anti-capital punishment, and antituition), conservative (antiaffirmative action, anti-nuclear freeze, pro-capital punishment, and protuition), or neutral (neither favoring nor opposing these policy stands). High interjudge agreement existed $(r=.90)$.

Following Petty and Brock (1979, p. 202), we constructed a ratio balance index, which measures the evaluative consistency of the thoughts reported and controls for the total number of thoughts. This measure consists of the ratio of either the number of liberal or conservative thoughts (whichever is greater) to the total number of liberal, conservative, and neutral thoughts. The closer subjects' scores were to 1 on the index, the more evaluatively consistent were their thoughts on the issue.

\section{Results}

\section{Correlations Among Major Dependent Variables}

The correlations among the three Semantic Differential scales ranged from .46 to .84 . We therefore created a single index for each issue based on the mean of the three scales (estimated reliabilities of composite indices ranged from .75 to .93 ). The correlations among different issues were much lower (mean $r=.18$ ).

The measures of cognitive structure derived from the thought protocols were highly correlated. More integratively complex protocols had lower ratio balance indices, mean $r(322)=-.80$, $p<.0001$. The measures of cognitive content derived from the thought protocols-the number of liberal and conservative thoughts-were highly correlated with the Semantic Differential scales. The more conservative the thoughts, the more conservative were the attitude scale responses, mean $r(323)=-.68$, $p<.0001$; the more liberal the thoughts, the more liberal were the attitude scale responses, mean $r(323)=.64, p<.0001$. Somewhat unexpectedly, however, the number of conservative thoughts was correlated with integrative complexity, mean $r(323)=.34, p<.0001$, whereas the number of liberal thoughts was not, mean $r(322)=.07$. This finding reflects the fact that subjects reported 2.7 times more liberal than conservative thoughts. Conservative thoughts often functioned as qualifications to otherwise liberal trains of thought-qualifications that, by definition, increased integrative complexity scores.

\section{Cognitive Structural Indices: Testing the Self-Criticism and Bolstering Hypotheses}

We conducted a $2 \times 4 \times 4$ (Timing of Measures $\times$ Type of Accountability $\times$ Issue) multivariate analysis of variance of the 


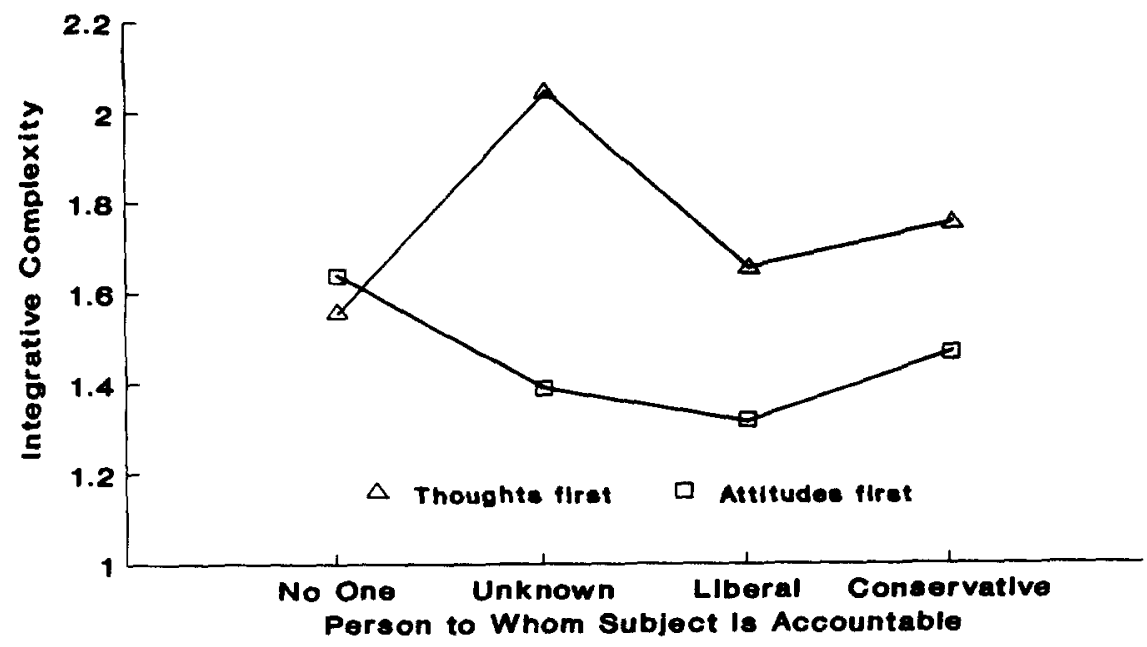

Figure 1. Integrative complexity as a function of accountability and timing-of-thought-assessment conditions.

integrative complexity and ratio balance measures. This analysis revealed a powerful interaction between the timing of the thought assessment and type of accountability on the cognitive structural indices, Rao's $F(6,155.5)=7.08, p<.001$. We conducted analyses of variance of the individual measures to explore the nature of this interaction.

Figure 1 and Table 1 present the mean integrative complexity of the thoughts that subjects reported. Two aspects of the interaction for this variable deserve special note. First, consistent with the preemptive self-criticism hypothesis of the social contingency model, subjects coped with accountability by thinking in more complex ways only when they reported their thoughts before responding to the attitude scales and when they did not know the views of the audience to whom they felt accountable. This trend, moreover, was significant in three of the four different issues on which subjects reported thoughts (with a trend in

Table 1

Mean Integrative Complexity and Ratio Balance on Social Issues

\begin{tabular}{lcc}
\hline Order and accountability condition & $\begin{array}{c}\text { Integrative } \\
\text { complexity } \\
\text { measure }^{\mathrm{a}}\end{array}$ & $\begin{array}{c}\text { Ratio } \\
\text { balance }^{\text {index }}\end{array}$ \\
\hline Thought listing before attitude commitment & & \\
Unaccountable & 1.56 & 0.82 \\
Accountable-unknown & 2.05 & 0.75 \\
Accountable-liberal & 1.66 & 0.82 \\
Accountable-conservative & 1.76 & 0.74 \\
Thought listing after attitude commitment & & \\
Unaccountable & 1.64 & 0.86 \\
Accountable-unknown & 1.39 & 0.88 \\
Accountable-liberal & 1.32 & 0.92 \\
Accountable-conservative & 1.47 & 0.90 \\
\hline
\end{tabular}

${ }^{a}$ Higher scores indicate more complex responses. Ratings ranged from 1 to $7 .{ }^{b}$ Higher scores indicate greater evaluative consistency on an issue. The index ranged from 0 to 1 . the predicted direction for the tuition issue). Focusing only on thoughts-first subjects, those accountable to an unknown individual were more integratively complex than unaccountable subjects, $F(1,80)=15.51, p<.001$; accountable-liberal subjects, $F(1,80)=10.48, p<.01$; and accountable-conservative subjects, $F(1,78)=5.21, p<.05$. Subjects in the thoughts-first and accountable-unknown condition also had higher complexity scores than subjects in any of the attitudes-first conditions, regardless of accountability condition: Their mean complexity score was .41 higher than that of unaccountable subjects, $F(1$, $78)=10.19, p<.001 ; .66$ higher than that of accountableunknown subjects, $F(1,78)=24.88, p<.001 ; .72$ higher than that of accountable-liberal subjects, $F(1,80)=39.66, p<.001$; and .58 higher than that of accountable-conservative subjects, $F(1,80)=21.58, p<.001$.

Second, attitudes-first subjects reported less complex thoughts than thoughts-first subjects $(M s=1.75$ vs. 1.45$), F(1$, $317)=30.59, p<.001$. Interestingly, contrary to the intrapsychic bolstering hypothesis of the social contingency model, the timing of the thought assessment was not sufficient to produce simplification of thought. Focusing only on unaccountable subjects, there was no difference in the complexity of thoughts that thoughts-first versus attitudes-first subjects reported $(M \mathrm{~s}=1.64$ vs. 1.56), $F(1,317)<1$. Consistent with the model, however, accountability lowered the complexity of reported thoughts across all four issues. The mean complexity for unaccountableattitudes-first subjects was 1.64 , which was 0.25 higher than for accountable-unknown subjects, $F(1,78)=11.43, p<.01 ; .32$ higher than for accountable-liberal subjects $F(1,78)=12.94$, $p<.001$; and 0.17 higher than for accountable-conservative subjects, $F(1,78)=2.70, p=.05$.

Table 1 also presents the results for the ratio balance index. Not surprisingly, in view of the powerful correlation between the two variables, these results are similar to those for integrative complexity, with two noteworthy exceptions. First, subjects accountable to a conservative audience reported as evaluatively inconsistent thoughts as subjects accountable to an unknown 
Table 2

Mean Number of Liberal and Conservative

Thoughts on Social Issues

\begin{tabular}{lcc}
\hline \multicolumn{1}{c}{ Order and accountability condition } & $\begin{array}{c}\text { Liberal } \\
\text { thoughts }\end{array}$ & $\begin{array}{c}\text { Conservative } \\
\text { thoughts }\end{array}$ \\
\hline Thought listing before attitude commitment & & \\
Unaccountable & 1.75 & 0.78 \\
Accountable-unknown & 1.64 & 0.89 \\
Accountable-liberal & 2.00 & 0.49 \\
Accountable-conservative & 1.49 & 0.93 \\
Thought listing after attitude commitment & & \\
Unaccountable & 2.26 & 0.71 \\
Accountable-unknown & 2.08 & 0.59 \\
Accountable-liberal & 2.02 & 0.79 \\
Accountable-conservative & 2.28 & 0.61 \\
\hline
\end{tabular}

Note. Number of liberal and conservative thoughts ranged from 0 to 5 .

audience in the thoughts-first conditions. The subjects in these two groups were, however, significantly more evaluatively inconsistent than subjects in any of the other conditions. Second, the impact for accountability in the attitudes-first conditions was less pronounced on the ratio balance index than it was on the integrative complexity measure. Accountability led attitudes-first subjects to report more evaluatively inconsistent thoughts than control subjects only in the accountable-liberal condition $(M \mathrm{~s}=.86$ vs. .92$), F(1,78)=3.84, p<.05$.

\section{Number of Liberal and Conservative Thoughts}

Table 2 presents the mean numbers of liberal and conservative thoughts. The results shed new light on the cognitive structural effects noted earlier. There was a substantial main effect for the timing of the thought listing. Attitudes-first subjects tended to bolster their attitudes. Because the predominant attitude in the subject pool was liberal, attitudes-first subjects reported more liberal thoughts than thoughts-first subjects $(M \mathbf{s}=$ 2.16 vs. 1.72$), F(1,316)=38.87, p<.0001$. Attitudes-first subjects also reported fewer conservative thoughts $(M \mathrm{~s}=.67 \mathrm{vs}$. $.77)$-an effect that, however, was not significant $(p>.20)$.

Further support for the bolstering hypothesis comes from comparing the correlations between attitudes and thoughts in the attitudes-first and thoughts-first conditions. As anticipated, the correlations between attitudes and thoughts were high in the thoughts-first conditions ( $r s=.60$ and -.58 for liberal and conservative thoughts, respectively), but were even higher in the attitudes-first conditons $(r s=.74$ and -.76 for liberal and conservative thoughts, respectively). Fisher's $r$ to $z$ transformation revealed the latter correlations to be significantly greater than the former (for liberal thoughts, $z=4.08, p<.0001$, and for conservative thoughts, $z=4.65, p<.0001$ ).

\section{Strategic Shifting of Public Attitudes}

Figure 2 and Table 3 present the mean liberalism-conservatism of subjects' attitudes. Subjects who knew the views of the prospective audience tended to report views compatible with those of the expected audience only in the thoughts-first condi- tions. These subjects reported more liberal attitudes to a liberal audience relative to the unaccountable controls $(M \mathrm{~s}=5.48 \mathrm{vs}$. $4.80), F(1,81)=11.93, p<.001$, and more conservative attitudes to a conservative audience relative to unaccountable controls $(M \mathrm{~s}=4.36$ vs. 4.80$), F(1,81)=7.53, p<.01$. Unexpectedly, the accountability effect fell far short of significance in the attitudes-first conditions, $F(3,159)=1.38, p<.25$, ns.

Including the numbers of liberal and conservative private thoughts as covariates attenuated but did not eliminate the effects of accountability to a liberal or conservative audience on public attitudes (both $p s<.05$ ). The interaction between accountability and timing of the thought listing did, however, cease to be significant, $F(1,314)=1.64, n s$. The number of liberal and conservative thoughts were highly significant covariates, $F(1,314)=113.6, p<.0001$, and $F(1,314)=110.7, p<$ .0001 , respectively.

\section{Effects of Anticipated Disagreement}

Previous work suggests that subjects who resist shifting toward the prospective audience interpret issues in more integratively complex and evaluatively inconsistent ways than subjects who shift (Tetlock, 1983a). In this study, subjects taking liberal positions in the accountable-conservative condition were not more complex or evaluatively inconsistent than subjects taking conservative positions (average $r=-.01$ ). As in previous work, however, subjects taking liberal positions in the accountableliberal condition were less complex than more conservative subjects (average $r=.24, p<.01$ ), and more evaluatively consistent (average $r=.36, p<.001$ ).

\section{Personality Correlates}

Exploratory tests of the effects of personality variables on integrative complexity yielded dogmatism as the only significant covariate. A repeated-measures analysis of covariance (ANCOVA) revealed that more dogmatic subjects tended to be less complex, $F(1,311)=3.76, p<.05 ; r(318)=-.21$. Controlling for dogmatism did not, however, alter any of the earlier results. Dogmatism also did not predict individual differences within experimental conditions in the expected manner. Dogmatic subjects were neither more prone to be self-critical in the thoughts-first, accountable-unknown condition nor more prone to be defensive in the attitudes-first conditions.

Social anxiety failed to play its hypothesized role in moderating strategic attitude shifts $(r<<.10)$. Social anxiety did, however, play its predicted role in moderating preemptive self-criticism in the thoughts-first, accountable-unknown condition. High social anxiety subjects reported more integratively complex thoughts, $r(38)=-.38, p<.05$. High social anxiety subjects were also more likely to engage in defensive bolstering of their attitudes in the attitudes-first, accountable-conservative condition. Here, high social anxiety subjects reported less integratively complex thoughts, $r(39)=-.46, p<.01$, and more evaluatively consistent thoughts, $r(39)=.50, p<.01$.

No other personality predictions received support.

\section{Phenomenology of Accountability}

We have focused on the private cognitive and public attitudinal strategies of coping with accountability, but we have largely 


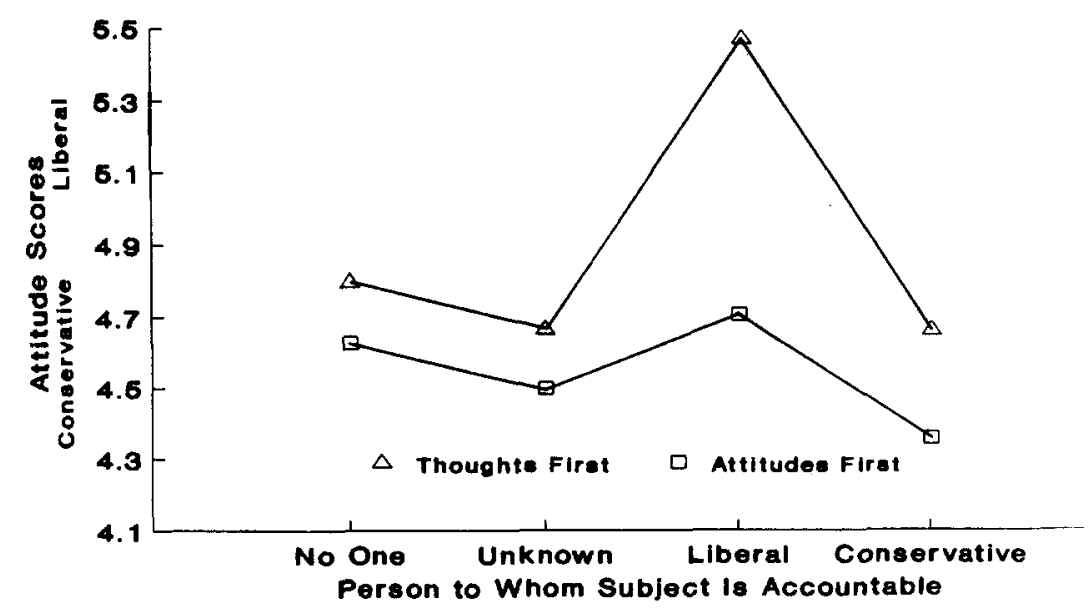

Figure 2. Mean liberalism-conservatism of attitudes as a function of accountability and timing-of-thought-assessment conditions.

ignored the experiential correlates of this social condition. Accountable subjects reported an interesting mix of emotional reactions. On the one hand, accountable subjects, regardless of the timing of the thought listing or the views of the audience, tended to feel more nervous $(M s=4.30$ vs. 3.11$), F(1,317)=$ $33.54, p<.01$; more embarrassed $(M s=3.85$ vs. 3.41$), F(1$, $371)=6.46, p<.01$; and more uncomfortable with the experiment $(M s=4.26$ vs. 3.15$), F(1,317)=32.57, p<.01$, than unaccountable subjects. On the other hand, accountable subjects also reported that they liked the experiment more $(M \mathrm{~s}=$ 4.16 vs. 3.37$), F(1,317)=21.01, p<.01$; and had more fun as a result of participating $(M s=3.93$ vs. 3.15$), F(1,317)=20.48$, $p<.01$, than their unaccountable counterparts. Accountability, in short, focused attention on the task at hand, inducing both negative and positive emotions.

\section{Discussion}

The data supported the major predictions of the social contingency model of judgment and choice. The model identified

Table 3

Mean Attitudes on Social Issues

\begin{tabular}{lc}
\hline Order and accountability condition & $\begin{array}{c}\text { Overall } \\
\text { liberalism- } \\
\text { conservatism }\end{array}$ \\
\hline Thought listing before attitude commitment & \\
Unaccountable & 4.80 \\
Accountable-unknown & 4.68 \\
Accountable-liberal & 5.49 \\
Accountable-conservative & 4.37 \\
Thought listing after attitude commitment & \\
Unaccountable & 4.64 \\
Accountable-unknown & 4.50 \\
Accountable-liberal & 4.71 \\
Accountable-conservative & 4.67 \\
\hline
\end{tabular}

Note. Higher scores indicate a more liberal attitude across the four issues. Scores ranged from 1 to 7. situational variables that activate each of three qualitatively distinct strategies of coping with accountability demands: strategic attitude shifts, preemptive self-criticism, and defensive bolstering. We found evidence of each coping strategy. Thoughts-first subjects who knew the views of the audience coped by shifting their public attitudes toward those of the anticipated audience. They expressed more liberal views to the liberal audience and more conservative views to the conservative audience. Thoughts-first subjects who did not know the views of the audience coped by thinking about issues in more integratively complex and evaluatively inconsistent ways. These subjects appeared to engage in preemptive self-criticism in which they tried to anticipate the various objections that potential critics could raise to the positions they were about to take (e.g., I may favor capital punishment, but I understand the opposing arguments). Finally, attitudes-first subjects coped in almost exactly the opposite way. These subjects responded to accountability by thinking about issues in less integratively complex ways. Far from engaging in self-criticism, these subjects were concerned with self-justification-with thinking of as many reasons as they could for why they were right and potential critics were wrong.

Some unexpected findings did, however, also emerge. One surprise was the disappearance of strategic attitude shifts in the attitudes-first conditions. Subjects moved their views toward the anticipated audience only when they had an extended opportunity to think about each issue. Attitude shifts, at least in this study, were largely cognitively mediated. This conclusion receives further support from the fact that attitude shifts in response to accountability were substantially reduced when we controlled-through ANCOVAs-for the number of liberal and conservative thoughts.

These results suggest that strategic attitude shifts are not always the product of heuristic or peripheral information processing (cf. Chaiken, 1980; Petty \& Cacioppo, 1986). If subjects were automatically or unthinkingly shifting their views toward the anticipated audience, subjects should have shifted to approximately the same degree in the thoughts-first and attitudesfirst conditions. The evidence suggests that the attitude shifts 
here were the product of systematic or central information processing-empathic efforts by subjects to understand the political perspective of the prospective discussion partner and to incorporate that perspective into their own view of the issue.

Another interesting finding was the absence of cognitive simplification effects in the attitudes-first, no-accountability condition. Making a private commitment to policy positions was not sufficient to induce less integratively complex and more evaluatively consistent thought. An additional condition had to be satisfied. Attitudes-first subjects reported less complex and more consistent thoughts only when they felt accountable for their views. These results can be assimilated into either an intrapsychic framework (e.g., expectation of future interaction induces self-awareness, which in turn sensitizes subjects to dissonant relations among cognitions) or an impression management framework (e.g., subjects attempt to prepare for the discussion by generating cognitions that make their positions appear as sound and justified as possible). These two levels of explanation are better viewed as complementary and mutually reinforcing than as contradictory (Tetlock \& Manstead, 1985).

A third, unexpected, finding was the generally weak performance of the personality variables measured here. The solealbeit partial-exceptions were the measures of dogmatism and social anxiety. More dogmatic subjects reported less complex thoughts-a finding consistent with early work on personality correlates of integrative complexity (Schroder, Driver, \& Streufert, 1967). High social anxiety subjects were more prone to complex, self-critical patterns of thinking in the thoughts-first, accountable-unknown condition and more prone to simple, self-justificatory patterns of thinking in the attitudes-first, accountable-conservative condition. Most predictions, however, were not supported. Dogmatism did not moderate cognitive coping responses to accountability (self-criticism vs. bolstering) and social anxiety did not moderate interpersonal coping responses to accountability (strategic attitude shifting). If personality variables do play moderator variable roles of this sort, it seems necessary to use more powerful research designs that build in low and high scores on personality variables as classification factors rather than probing for relations through analyses of within-cell correlations.

One should also keep in mind the prevailing political mood within the subject population. Subjects generally endorsed liberal positions and reported more liberal than conservative thoughts. This atmosphere probably reduced willingness to shift political attitudes to the right in the accountable-conservative condition. Moreover, not only were subjects less likely to shift toward the conservative audience, those who resisted shifting in this condition were less likely to engage in preemptive self-criticism in their thought protocols than were subjects who expressed conservative views to a liberal audience. Liberals facing a conservative were, in short, less self-critical than conservatives facing a liberal-a result that is more parsimoniously attributed to the predominantly liberal normative environment surrounding the study than it is to ideological differences in cognitive style (Tetlock, 1984).

In addition to the specific findings reported here, the concept of accountability itself requires some clarification. Accountability bears a marked theoretical resemblance to experimental manipulations used in other lines of research, including work on cognitive tuning (Zajonc, 1960), self-awareness (Carver \& Scheier, 1987), and consequentiality (McAllister, Mitchell, \& Beach, 1979). It is reasonable to ask whether similar processes underlie the effects of these different empirical operations. Research to date, however, underscores the need to make careful methodological and theoretical distinctions. For instance, Zajonc's "transmission-set" manipulation led subjects to expect to communicate their views to a third party with no independent knowledge of the target issue or person; this situation is very different from expecting to justify (not just communicate) one's views to an audience who may know as much as, or more than, the subject does about the issues. Cognitive tuning manipulations also turn out to have very different effects from accountability. Inducing a transmission set magnifies the overattribution effect, whereas accountability reduces it (Tetlock, 1985b).

In a similar vein, accountability is similar to but also different from manipulations of consequentiality or the importance or personal relevance of the issue. Accountability and consequentiality manipulations sometimes have similar effects (e.g., McAllister et al., 1979), but not always. Increasing the importance of the judgment task has been found, for example, to exacerbate overconfidence, whereas accountability reduces the same effect (Tetlock \& Kim, 1987).

Accountability manipulations are also similar to self-awareness manipulations in important ways (Carver \& Scheier, 1987). To create credible accountability manipulations, we have found it necessary to tell subjects that the communication phase of the experiment will be tape recorded (a common method of inducing public self-awareness). One could argue, and it would be consistent with the spirit of the social contingency model, that the effects of self-awareness are partly mediated by heightened public self-awareness-where public self-awareness is defined in the classic symbolic interactionist sense of taking the perspective of others toward one's own attitudes and behavior. Public self-awareness theory is not well positioned, however, to explain the pattern of data here. The self-awareness component of the accountability manipulation was a constant that did not vary with the views of the prospective audience or the timing of the thought listing. It would be difficult, therefore, for selfawareness theory to explain the rather complex interactions between types of accountability and timing of measures that were repeatedly observed across issues in this study.

Finally, we should comment on the broader theoretical implications of our findings. The predictions of the social contingency model of judgment and choice generally held up well. Theories of social cognition need to take explicit account of how interpersonal and institutional demands for accountability shape not only the cognitive content but also the underlying cognitive structure of the attitudinal stands that people take. Accountability demands can motivate people to be either more flexible multidimensional information processors or more rigid, evaluatively consistent information processors. These different patterns of thinking serve different functions in different social situations. Precommitment cognitive preparation for discussion appears to be largely an exercise in role taking - a finding consistent with the symbolic interactionist theory (e.g., Stryker \& Statham, 1985). This preparation leads subjects accountable to an audience with known views to give more 
careful consideration to that perspective and to shift their public stands in that direction as well. It leads subjects accountable to an unknown audience to anticipate counterarguments that critics from either side of the political spectrum might raise and to incorporate those positions into balanced, middle-ground policy stands. Postcommitment cognitive preparation for discussion appears largely to be an exercise in intellectual self-defense-the generation of justifications for positions taken. As a result, the thoughts reported tend to be both integratively simple and evaluatively consistent.

In brief, a robust, replicable, and theoretically interpretable pattern emerges in this study. Interpersonal goals and concerns play a key role in shaping the underlying cognitive structure of expressed political attitudes. How people think depends in part on why people think-a conclusion strikingly reminiscent of the early functionalist theories of attitudes.

\section{References}

Buss, A. H. (1980). Self-consciousness. San Francisco: Freeman.

Carver, C., \& Scheier, M. (1987). The blind men and the elephant: Selective examination of the public-private literature gives rise to a faulty perception. Journal of Personality, 55, 525-541.

Chaiken, S. (1980). Heuristic versus systematic information processing and the use of source versus message and cues in persuasion. Journal of Personality and Social Psychology, 39, 752-766.

Cialdini, R. B., Levy, A., Herman, C. P., \& Evenbeck, S. (1973). Attitudinal politics: The strategy of moderation. Journal of Personality and Social Psychology, 25, 100-108.

Cvetkovich, G. (1978). Cognitive accommodation, language, and social responsibility. Social Psychology Quarterly, 41, 149-155.

Fiske, S. T., \& Taylor, S. (1984). Social cognition. Reading, MA: Addison-Wesley.

Fox, F., \& Staw, B. M. (1979). The trapped administrator: The effects of job insecurity and policy resistance upon commitment to a course of action. Administrative Science Quarterly, 24, 449-471.

Gergen, K. J. (1982). Toward transformation in social knowledge. New York: Springer-Verlag.

Hagafors, R., \& Brehmer, B. (1983). Does having to justify one's decisions change the nature of the judgment process? Organizational Behavior and Human Performance, 31, 223-232.

Jones, E. E., \& Wortman, C. (1973). Ingratiation: An attributional approach. Morristown, NJ: General Learning Press.

McAllister, P. W., Mitchell, T. R., \& Beach, L. R. (1979). The contingency model for the selection of decision strategies: An empirical test of the effects of significance, accountability, and reversibility. Organizational Behavior and Human Performance, 24, 228-244.

Petty, R. E., \& Brock, T. C. (1979). Effects of Barnum personality assessments on cognitive behavior. Journal of Consulting and Clinical Psychology, 47, 201-203.

Petty, R. E., \& Cacioppo, J. T. (1986). The elaboration-likelihood model of persuasion. In L. Berkowitz (Ed.), Advances in experimental social psychology (Vol. 16, pp. 123-205). New York: Academic Press.

Pruitt, D. (1981). Negotiation behavior. New York: Academic Press.

Rokeach, M. (1960). The open and closed mind. New York: Basic Books.

Rozelle, R. M., \& Baxter, J. C. (1981). Influence of role pressures on the perceiver: Judgments of videotaped interviews varying judge accountability and responsibility. Journal of Applied Psychology, 66, 437-441.

Sampson, E. E. (1981). Cognitive psychology as ideology. American Psychologist, 36, 730-743.
Schlenker, B. R. (1982). Translating actions into attitudes: An identityanalytic approach to the explanation of social conduct. In L. Berkowitz (Ed.), Advances in experimental social psychology (Vol. 15, pp. 186-224). New York: Academic Press.

Schroder, H. M., Driver, M., \& Streufert, S. (1967). Human information processing. New York: Holt, Rinehart \& Winston.

Scott, M., \& Lyman, S. (1968). Accounts. American Sociological Review, 33, 46-62.

Sherman, S. J., \& Corty, E. (1984). Cognitive heuristics. In R. S. Wyer \& T. K. Srull (Eds.), Handbook of social cognition (Vol. 1, pp. 189282). Hillsdale, NJ: Erlbaum.

Staw, B. M. (1980). Rationality and justification in organizational life. In B. M. Staw \& L. Cummings (Eds.), Research in organizational behavior (Vol. 2, 1-57). Greenwich, CT: JAI Press.

Stryker, S., \& Statham, A. (1985). Symbolic interaction and role theory. In E. Aronson \& G. Linzey (Eds.), Handbook of social psychology (Vol. 1, pp. 311-378). Hillsdale, NJ: Erlbaum.

Tetlock, P. E. (1979). Identifying victims of groupthink from public statements of decision makers. Journal of Personality and Social Psychology, 37, 1314-1324.

Tetlock, P. E. (1981a). The influence of self-presentational goals on attributional reports. Social Psychology Quarterly, 41, 300-311.

Tetlock, P. E. (1981b). Pre- to post-election shifts in presidential rhetoric: Impression management or cognitive adjustment? Journal of Personality and Social Psychology, 41, 207-212.

Tetlock, P. E. (1983a). Accountability and complexity of thought. Journal of Personality and Social Psychology, 45, 74-83.

Tetlock, P. E. (1983b). Accountability and the perseverance of first impressions. Social Psychology Quarterly, 46, 285-292.

Tetlock, P. E. (1984). Cognitive style and political belief systems in the British House of Commons. Journal of Personality and Social Psychology, 46, 365-375.

Tetlock, P. E. (1985a). Accountability: The neglected social context of judgment and choice. In B. Staw \& L. Cummings (Eds.), Research in organizational behavior (Vol. 1, pp. 297-332). Greenwich, CT: JAI Press.

Tetlock, P. E. (1985b). Accountability: A social check on the fundamental attribution error. Social Psychology Quarterly, 48, 227-236.

Tetlock, P. E. (1986). A value pluralism model of ideological reasoning. Journal of Personality and Social Psychology, 50, 819-827.

Tetlock, P. E., \& Boettger, R. (1989). Accountability: A social magnifier of the dilution effect. Journal of Personality and Social Psychology, 57, 388-398.

Tetlock, P. E., Hannum, K., \& Micheletti, P. (1984). Stability and change in senatorial debate: Testing the cognitive versus rhetorical style hypotheses. Journal of Personality and Social Psychology, 46, 979-990.

Tetlock, P. E., \& Kim, J. I. (1987). Accountability and judgment processes in a personality prediction task. Journal of Personality and Social Psychology, 52, 700-709.

Tetlock, P. E., \& Manstead, A. S. R. (1985). Impression management versus intrapsychic explanations in social psychology: A useful dichotomy? Psychological Review, 92, 67-82.

Wicklund, R. A., \& Brehm, J. W. (1976). Perspectives on cognitive dissonance. Hillsdale, $\mathrm{NJ}$ : Erlbaum.

Zajonc, R. B. (1960). The process of cognitive tuning in communications. Journal of Abnormal and Social Psychology, 61, 159-167.

Received September 20, 1988

Revision received May 15, 1989

Accepted May 16, 1989 